\title{
Good Laboratory Practice for Optimization
}

Research 
Graham Kendall ${ }^{1,2}$

and

Ruibin $\mathrm{Bai}^{3}$

and

Jacek Błazewicz ${ }^{4}$

and

Patrick De Causmaecker ${ }^{5}$

and

Michel Gendreau ${ }^{6}$

and

Robert John ${ }^{1}$

and

Jiawei $\mathbf{L i}^{1}$

and

Barry McCollum ${ }^{7}$

and

Erwin Pesch $^{8}$

and

Rong $\mathbf{Q u}{ }^{1}$

and

Nasser Sabar ${ }^{2}$

and

Greet Vanden Berghe ${ }^{5}$

and

Angelina $\mathrm{Yee}^{2}$

${ }^{1}$ ASAP Research Group, School of Computer Science,

University of Nottingham, UK

${ }^{2}$ University of Nottingham Malaysia Campus, Malaysia

${ }^{3}$ University of Nottingham Ningbo, Ningbo, China

${ }^{4}$ Institute of Computing Science, Poznan University of Technology

${ }^{5}$ Department of Computer Science, KU Leuven, Belgium

${ }^{6}$ University of Montreal, Montreal, Canada

${ }^{7}$ School of Electronics, Electrical Engineering and Computer Science, Queen's University Belfast, UK

${ }^{8}$ Department of Management Information Science, Universität Siegen

September 20, 2015 


\section{Abstract}

Good Laboratory Practice has been a part of non-clinical research for over 40 years yet. Optimization Research, despite having many papers discussing standards being published over the same period of time, has yet to embrace standards that underpin its research.

In this paper we argue the need to adopt standards in optimization research. Building on previous papers, many of which have suggested that the optimization research community should adopt certain standards, we suggest a concrete set of recommendations that the community should adopt.

We also discuss how the proposals in this paper could be progressed.

\section{Optimization, Operations Research, Reproducibility}

\section{Introduction}

As far back as (Ignizio, 1971), and perhaps before, there was discussion in the scientific literature as to how the scientific community should report algorithmic results. Looking at some recent papers, little seems to have changed and if we compare the optimization research community against other communities (e.g. non-clinical research) it is debatable whether it would stand up to close scrutiny with regard to the research methods that are employed. That is not to say that the research is not conducted without relevant integrity, but the scientific process, and the way that experiments are reported, could be subject to some criticism. It should also be noted that other disciplines are not without criticism ((Anon, 2013)).

The academic community is also aware that if we do archive our data and results then we run the risk of losing this data. The study in (Vines et al., 2013) shows that the availability of data in research papers is strongly affected by the age of the article. They conclude that data cannot be reliably preserved by individual researchers. The optimization research community should ensure that the data that underpins its research findings is available in a centralized location for future researchers, and we should not be reliant on single researchers or research groups.

Good Laboratory Practice (GLP) has been established for over forty years in non-clinical research and is used to assess the safety or efficacy of chemicals, including pharmaceuticals, to man, animals and the environment. It provides a quality system of management controls for research laboratories and organizations to ensure the uniformity, consistency, reliability, reproducibility, quality, and integrity of chemical research. Bioinformatics is an area where some of these practices have spread to optimization Research, due to the close relationship between biologists and optimization Research ((Błazewicz et al., 2005, Lukasiak et al., 2010)). As an example, Bioinformatics insists on computer code being made available, which is one of the areas discussed in this paper (see Section 4.12). 
Given its long history ((Gass and Assad, 2006)), dating back to positioning radar stations in World War II ((Brown, 1999)) it is, perhaps, surprising that optimization research has few documented quality standards that underpin at least some of its research. Non-clinical research, undoubtedly, has the potential to affect the lives of a great number of people; whether positively or adversely. The same is also true of optimization research. Perhaps even more so considering the scope of its reach. There are some classic areas which optimization researchers have embraced, and regularly report on. To give just a small sample, we provide this short list of a few representative optimization areas. We have identified papers from the following areas, both recent and from over 50 years ago, just to demonstrate how established some of them are.

- Assignment Problems - (Cattrysse and Van Wassenhove, 1992, Loiola et al., 2007, Pentico, 2007)

- Bioinformatics - (Błazewicz et al., 2005, Lukasiak et al., 2010)

- Forecasting - Fildes $(1979,1985)$, Fildes et al. (2008), Ghobbar and Friend (2003), Trapero et al. (2015)

- Job Shop Scheduling - (Błazewicz et al., 1996, 2007, Brucker et al., 2007, Cheng et al., 1999a,b, Kiran and Smith, 1984)

- Knapsack Problem - (Lin, 1998, Lust and Teghem, 2012, Pisinger, 2007, Salkin and Kluyver, 1975, Wilbaut et al., 2008)

- Personnel Scheduling - (Ernst et al., 2004, Miller, 1976)

- Sports Scheduling - (Kendall et al., 2010, Rasmussen and Trick, 2008)

- Traveling Salesman Problem - (Bellmore and Nemhauser, 1968, Burkard et al., 1998, Cook, 2011, Lust and Teghem, 2010)

- Vehicle Routing - (Archetti and Speranza, 2008, Braysy et al., 2004, Braysy and Gendreau, 2005a,b, Cordeau et al., 2002, Doerner and Schmid, 2010, Gendreau et al., 2008, Jaillet and Wagner, 2008, Laporte, 2009, Vidal et al., 2013)

Of course, there are many other areas which also come under the optimization research radar. Indeed, it is difficult to think of a discipline/sector that either does not currently use optimization research, or could benefit from some form of optimization. Any sector that ever use phrases such as minimize, maximize, reduce cost, increase profit, make more efficient, save time/money/energy etc. can benefit from the tools and methodologies available to optimization researchers.

Given the potential high impact and visibility of its research it is beholden on the community to ensure that the research it carries out is done to the highest possible standards, which would stand up to external scrutiny should that be required. There is no suggestion that any research that is currently conducted 
falls short of these standards. Indeed, as far as the authors are aware, our research is well respected and the peer review process is fair, stringent and works well.

In this paper, we propose a set of procedures that we hope will further enhance the quality of the research that the optimization research community carries out. If these procedures were adopted, it would ensure that different laboratories/researchers were working to the same minimum standards which would enhance the reproducibility of results, the comparison of results and the efficiency of individual researchers and teams. Moreover, we suggest that papers that adopt the standards presented in this paper would be able identify this in the paper, which would provide a further indication of the paper's quality.

The recommendations in this paper are applicable to most, if not all, areas of optimization, where simulations are carried out, whether that is exact methods, heuristics, meta-heuristics or hyper-heuristics. These algorithms, especially where multiple computational experiments are required, often require some form of statistical analysis, so we need to ensure that this element of the algorithm design/reporting is handled appropriately. Many domains also have benchmark datasets, providing opportunities for further standards to be adopted.

For completeness, we provide the following definitions of the main areas that are covered by the recommendations in this paper. These definitions are mostly drawn from the recent scientific literature and are representative, rather than being definitive, as there are no widely accepted definitions for these terms.

Exact Optimization Methods ((Jourdan et al., 2009)): "Exact methods find the optimal solution and assess its optimality."

Heuristics ((Zanakis et al., 1989)): "Their [heuristics] appeal stems from their ability to produce quickly near-optimal solutions to difficult optimization problems. As opposed to the advanced mathematics that is required to develop theoretical results in the optimization arena, the development of heuristics is chiefly an art and a creative problem solving endeavor" and "Construction algorithms generate a solution by adding individual components (e.g., nodes, arcs, variables) one at a time until a feasible solution is obtained. Greedy' algorithms, seeking to maximize improvement at each step, comprise a large class of construction heuristics. In most construction heuristics, a feasible solution is not found until the end of the procedure" and "Improvement heuristics begin with a feasible solution and successively improve it by a sequence of exchanges or mergers in a local search. Generally, a feasible solution is maintained throughout the procedure."

Metaheuristics ((Sörensen and Glover, 2013, Talbi, 2009)): "A metaheuristic is a high-level problem-independent algorithmic framework that provides a set of guidelines or strategies to develop heuristic optimization algorithms. The term is also used to refer to a problem-specific implementation of a heuristic optimization algorithm according to the guidelines expressed in such a framework."

Hyper-Heuristics ((Burke et al., 2013)): "A search method or learning mechanism for selecting or generating heuristics to solve computational search problems." 
In this paper, we provide a set of suggested standards that optimization laboratories/researchers, might want to adopt. The recommendations may require some refining, which is why we have provided a suggested name (Good Laboratory Practice for Optimization Research (GLP4OPT)), along with a version control mechanism so that it is clear what standards are being adopted at any given time. The version we are proposing in this paper is version GLP4OPT version 1.00 .

\section{Related Work}

\subsection{Optimization Research}

One of the earliest papers that looked at comparing computational methods can be found in (Hoffman et al., 1953), where they compared several types of linear program solvers, concluding that the simplex method was the best from three examined. It is interesting to note that some of the discussion was about preprocessing from magnetic tapes and the effect this has on the overall computation time. We do not have to consider these type of devices today, but it is interesting to note that researchers hardly ever mention the overheads of certain devices these days.

In ((Ignizio, 1971)) there were calls for establishing standards for comparing algorithms. Ignizio proposed a standard reporting format and measurement standards. In its summary, the author says "This author ... believes that our profession needs to establish such standards as previously discussed. By establishing such standards we can expect to (1) upgrade the status of our profession, (2) eliminate many marginal publications of dubious value, and (3) provide a means by which algorithms may be selected more objectively by the practitioner." We would suggest that over 40 years on, that this is still an aspiration for the optimization research community.

Another call was made for guidelines in 1978 by (Jackson and Mulvey, 1978). They said that "Unfortunately, the methodology for conducting such computational experiments has not received systematic study, and no set of generally accepted guidelines has been available." They surveyed fifty papers, reporting how they have reported their experimental results. Their concluding remarks include "This paper shows that a consensus for conducting computational experiments has not been reached, although patterns can be detected within certain areas of mathematical programs. On the whole, the more recent experiments appear improved in methodology only slightly over their predecessors." Over 30 years later (Sörensen, 2015) makes similar comments with regard to taking up guidelines - "By setting up a statistical experiment, the main and interaction effects of the different algorithmic parameters on the solution quality produced by the meta-heuristic can be determined in a statistically valid way, and the optimal combination of parameter levels can be determined. Methods and guidelines to perform this step in the algorithm design are readily available ((Adenso-Diaz 
and Laguna, 2006, Coy et al., 2001)), but have not caught on in any significant way."

In August 1973 this topic was discussed by the Mathematical Programming community when it was raised at the Stanford Mathematical Programming Symposium ((Balinski, 1978)). In an editorial note, (Balinski, 1978) noted that the paper by (Crowder et al., 1978) would promote further discussion, which might eventually lead to a formal position being taken by the Committee on Algorithms. Balinski further notes that flexible editorial policy should still be used and any guidelines should not be used as a way to simply reject a paper.

The paper referred to by Balinski ((Crowder et al., 1978)) presents a set of recommended standards for presenting computational experiments from mathematical programming. Their opening remarks state that a review of papers in the area show a high level of mathematical expertise from the authors but this does not translate into the rigor that would be expected in order to reproduce the experiments being reported, for example, important parameters are often omitted. Crowder et al. further say that the mathematical programming community does not compare well to social science research in their scientific rigor. They state that part of the problem is that there are no published standards and see their paper going some way toward resolving this issue. The paper specifically discusses experimental design, reporting of computational results and suggestions such as how to present an algorithm. They conclude the paper with a checklist of important points.

Whilst recognizing that it is almost impossible to totally reproduce a given experiment, due to technological changes, it should be possible to produce the results within some tolerance, recognizing the technological changes that have taken place. Crowder et al. further note that "It is here where editors are required to exercise their judgment on whether sufficient evidence has been provided, to convince them that the criterion of reproduceability could be met if tested". Moreover, the authors stress, "However, an absolute, reasonable, and scientifically justifiable criterion should be that the authors themselves be able to replicate their experiment. This is one of the basic principles of the scientific method and should be actively pursued in any properly conducted scientific inquiry." It is also interesting to note that they suggest that two versions of the paper should be submitted. The first will be published in the journal. The second provides all the details that may not be appropriate for the published article. Given that the internet has advanced so much since 1978, it would be much easier to meet this aspiration by the use of supplementary files.

The same authors published a related paper the following year ((Crowder et al., 1979)), which covered a lot of similar material, including a checklist of important points to consider when evaluating or reporting computational experiments. These were split into recommended and optional points, and included presenting a complete description of the algorithm; the programming language and compiler used, along with the options that were set; the computer environment; a description of any special data structures that were utilized; a description of the input data; the use of standard test problems and reporting of the results; a clear statement of the objectives of the experiment which can be 
evaluated by the reviewers and a complete description of the problem generator.

(Barr et al., 1995), in the first issue of the Journal of Heuristics, discussed how to design and report computational experiments. This is a thought provoking paper, presenting some historical readings which discuss how mathematical programming methodologies should be compared ((Crowder et al., 1979)). In our view, this paper is essential reading for anybody who has an interest in this area. Barr et al.'s paper could be seen as being critical of the heuristics/metaheuristics community. It was actually providing some suggestions as to how the community could be more rigorous in reporting its experiments and findings.

Jackson et al. (1991) say that "Controversy often surrounds the reporting of results from scientific experimentation. Subtle points with unrecognized but profound effects are sometimes overlooked, and testing procedures are sometimes inadequate." Their report does not propose a new set of guidelines but rather "We have two goals: (1) to present a concise set of principles to guide authors, editors, and referees in assessing computational tests, and (2) to clarify existing guidelines in certain areas." Their report builds on the work of (Crowder et al., 1979), using this as the base set of guidelines. The authors say that it is not possible to provide a complete set of guidelines but, instead, to provide a set of principles. These being, "(1) the results presented must be sufficient to justify the claims made; (2) there must be sufficient detail to allow reproducibility of the results; and (3) it is not the referee's duty to reproduce the results."

Golden and Stewart (1985) consider ways that statistical analysis can be carried out on the TSP (Traveling Salesman Problems). Although focusing on the TSP (as that is the subject of the book from which this chapter is taken from) the discussion on statistical tests and how to apply them is valuable. A worked example of applying the Wilcoxon signed rank test and the Friedman test demonstrates how three heuristics can be compared, rather than just making an assumption that one of the heuristic dominates the other two. Limitations of using these statistical tests are given, suggesting that an expected utility function is used instead. This is simple to apply but does rely on some arbitrary assumptions.

The following year (Golden et al., 1986) published an article that looked at the experimentation issues in optimization. The paper focuses on three case studies, with an initial discussion on the statistical analysis. It is interesting to note that one of the case studies considers simulated annealing ((Kirkpatrick et al., 1983)), which is one of the first times that a meta-heuristic is discussed in this context, due to its (then) recent introduction.

Greenberg (1990) considers how and why to perform computational testing and how much testing should be conducted. The paper also addresses the issue of when commercial confidentiality is claimed. The author's view is that if a research contribution is being claimed then "Any research paper whose merit depends critically (perhaps decisively) on the computational results must be prepared to have the results reviewed by referees." Later, this point is reemphasized - "Under no circumstances will a paper be published in the ORSA Journal on Computing whose merit and information content depend critically on empirical claims that are not subjected to such review.' 
Greenberg (1990) was written from the perspective of a specific journal. IIE Transactions also published its own guidelines when reporting computational results ((Lee et al., 1993)). Topics covered include comparison with existing methods, performance analysis, reproducibility and complexity analysis.

Hooker wrote two articles in the mid-nineties ((Hooker, 1994, 1995)). In his 1994 paper, he called for the need to have an empirical science of algorithms. He argues for rigorous experimental design and analysis and also for the development of empirically-based explanation theories. An analytical approach to algorithm analysis has, he argues, turned into a science, whereas empirical testing has not. His concerns are expressed as follows "Computational experiments are widely reported in scholarly publications. But these efforts fall short of science on several levels. To begin, the testing is usually quite informal, at least in the optimization research literature. One occasionally sees tests conducted according to the principles of experimental design, or results analyzed using rigorous statistical methods. But only occasionally. Not even minimal standards of reproducibility are observed by most authors." He goes onto to say that as a discipline, we do not encourage the publishing of negative results, which could be as important as a positive result when testing a given hypothesis. In his 1995 paper, Hooker argues that a more scientific approach is required in controlled experimentation. He likens the current state of play as resembling a track meet, where the aim is simply to beat the opponent. If the selected algorithm beats all other algorithms on selected test cases, it is submitted for publication, else the research is written off as a failure and the next idea is attempted. One section of the paper is devoted to the "evils of competitive testing", outlining the problems it presents (e.g. different levels of coding skills, different machines being used, the problems of using benchmark instances etc.). He goes onto say how all these issues can be removed, but it would need support and recognition from the scientific community.

McGeoch (1996) also makes the case for a more scientific way to study algorithms. One-dimensional bin packing is used as an example application, in order to discuss the issues addressed in the paper. Issues specifically addressed include planning the experiments, conducting a pilot study, developing and running experiments and statistics and data analysis.

Ahuja and Orlin (1996) discuss the use of CPU time as a measure of performance. They suggest that this is too implementation dependent and suggest that researchers should use representative operation counts, being a fairer way to provide comparative analysis.

Rardin and Uzsoy (2001) provide a tutorial of carrying out heuristic optimization experiments. This paper is also essential reading for those interested in this area. The paper focuses "squarely on empirical evaluation of heuristic optimization methods." They acknowledge that "the questions are difficult, and there are no clear right answers", seeking "only to highlight the main issues and present a number of alternative ways of addressing them under different circumstances, as well as a number of pitfalls to avoid." Among the topics discussed in the paper are research vs development, designing computational experiments, sources of test instances (real world, random variants of real world instances, 
libraries of instances, random generated instances) and measuring performance, carrying out analysis and presenting results. They also present a case study, based on one machine scheduling.

A more recent paper ((Sörensen, 2015)) is certainly critical of this community. In his opening, the author says "In this paper, we will argue that this line of research [the proliferation of meta-heuristic methodologies] is threatening to lead the area of meta-heuristics away from scientific rigor". In the concluding remarks, it states "Although several authors have developed procedures to make a statistically sound comparison (see, e.g., (Barr et al., 1995, Rardin and Uzsoy, 2001)), widespread acceptance of such procedures is lacking. Perhaps a set of tools is needed, i.e., a collection of statistical programs or libraries specifically designed to determine the relative quality of a set of algorithms on a set of problem instances. These should both be easy to use, and their results should be easy to interpret. Until such tools are available and a specific comparison protocol is enforced by journal editors and reviewers, the door is left open for researchers to select the method of comparison that proves the point it is intended to prove". A related theme can be found in (Hooker, 2007) who argues that a bad future for Constraint Programming and Operations Research is one "which is defined by the computational techniques, rather than by the phenomena they study", later saying that operations research has remained strong for the past fifty years despite the fact that many new methodologies have been introduced.

A very recent paper (Boylan et al., 2015) investigated replicability and reproducibility in forecasting. The study took an important paper (Miller and Williams, 2003), which had won an outstanding paper award and is highly cited, and asked two independent research teams to replicate the results. They encountered several difficulties, including data clarification, method clarification, using different software and accuracy measures. The two teams reached almost the same results as the original paper, but they were different and the teams would not have arrived at the same conclusions as Miller and Williams.

We actually started writing this paper before being aware of papers such as (Boylan et al., 2015, Rardin and Uzsoy, 2001, Sörensen, 2015), but if we had read those papers before conducting our literature survey, it would certainly have motivated us to write this paper. Many of the ideas expressed by the authors we cite above are captured in the ideas we present later in this paper, by way of a number of recommendations.

We have gone one stage further than previous authors by providing an explicit set of recommendations that we hope will form the basis as a set of guidelines for the community to adopt. Non-clinical researchers abide by the Good Laboratory Practice guidelines. The meta-heuristic community, we believe, needs such practices, many of which have been presented by other authors, but there is a need for the community to embrace and use them, rather than just discuss them every few years. We hope that the presentation as a set of recommendations will provoke this discussion, leading to an adoption of the recommendations (or a version of them) to be the guiding principles by which we carry out optimization research. 


\subsection{Other Disciplines}

Other disciplines are not without their faults.

In the medical area (Ioannidis, 2005) considered 45 highly cited papers, finding that seven (16\%) were later contradicted and another seven had reported effects that were stronger than those reported in later studies. Twenty $(44 \%)$ have been replicated in later studies with the remaining eleven (24\%) not having been challenged to date.

In forecasting, Evanschitzky and Armstrong (2010) studied the replications that were carried across two forecasting journals between 1996 to 2008. They found that just over a third (35.3\%) of the replications confirmed the results of the initial study, $45.1 \%$ provided partial support and $19.6 \%$ provided no support. Given that about one fifth of replication studies do not provide support for the original study Evanschitzky and Armstrong suggest that there is a need for more replication studies. They also say that "journals can aid this by requiring and archiving full disclosure details and by inviting authors to replicate specific papers."

A study going back to 1994 (Hubbard and Armstrong, 1994) showed that of 1,120 papers sampled none were replications of previous work. Twenty of the papers were extensions, with twelve of those conflicting with the original work and only three confirming the previous work. This study was extended in 2007 (Evanschitzky et al., 2007), with the authors noting that the editorial policies of some journals now encourage replication. However, they note "Results show that the replication rate has fallen to 1.2\%, a decrease in the rate by half. As things now stand, practitioners should be skeptical about using the results published in marketing journals as hardly any of them have been successfully replicated."

In the disciplines of Accounting, Economics, Finance, Management and Marketing Hubbard and Vetter (1996) carried out an an analysis of 18 business journals, covering 1970-1996. It showed that replication studies are not common in the business disciplines but when they are carried out the results are often at odds with the original study.

Easley et al. (2000) say that "The role of replication in marketing research has been a tenuous one at best." They argue that the absence of replication research in the social sciences is the result of incorrect perceptions about how the studies should be carried out and how acceptable these studies would be.

Nature has recently published a series of articles around the idea of Reducing our irreproducibility ((Anon, 2013) $)^{1}$. One of the articles focuses on the importance of reproducibility ((Russell, 2013)), arguing that grant funding should be tied to be able to reproduce results, presenting a series of counter arguments against what would be seen as an unpopular proposal by some in the community. (Vaux, 2012) argues that many papers contain incorrect or sloppy statistics, which leads to sloppy science. The paper also serves as a good point of reference for a general introduction to statistics and we note that there are some excellent papers in the Operations Research community which outline

\footnotetext{
${ }^{1}$ The full series of articles are freely available at go.nature.com/huhbyr
} 
various statistical tests and how they can be used (for example, (Taillard et al., 2008)).

Begley and Ellis (2012) argues that standards need to be raised in pre-clinical trials for cancer research. They report that only $25 \%$ of published pre-clinical trials could be validated to the point at which projects could continue. Perhaps, more worrying, is that the irreproducible papers had spawned many other papers that were based on the original findings. In order to counter such issues, (Baker, 2012) says that some scientific publishers are supporting an initiative, which would request high profile authors to have the results verified by an independent laboratory. (Prinz et al., 2011) says that many pharmaceutical companies run in house validation programmes before making a significant investment. However, they often find that exciting results in a scientific paper are difficult to reproduce. The paper concludes that literature data on potential drugs should be viewed with caution and the importance of carrying out a verification test.

\subsection{Good Laboratory Practice (GLP)}

An internationally recognized definition of GLP is available on the The Medicines and Healthcare Products Regulatory Agency (MHRA) web site. This organization carries the responsibility for regulating all medicines and medical devices in the UK.

"Good Laboratory Practice (GLP) embodies a set of principles that provides a framework within which studies are planned, performed, monitored, recorded, reported and archived. These studies are undertaken to generate data by which the hazards and risks to users, consumers and third parties, including the environment, can be assessed for pharmaceuticals, agrochemicals, veterinary medicines, industrial chemicals, cosmetics, food and feed additives and biocides. GLP helps assure regulatory authorities that the data submitted are a true reflection of the results obtained during the study and can therefore be relied upon when making risk/safety assessments." 2

GLP was established following four scientists being put on trial after faking drugs and chemical studies during the 1970's ((Marshall, 1983)). GLP was introduced in 1972 in New Zealand and Denmark and then in 1978 in the USA. The principles were later adopted by the Organization for Economic Co-operation and Development (OECD) in 1992. The OECD has helped to promote the principles in many countries since that time.

\section{Good Laboratory Practice for Optimization Research - GLP4OPT}

In this section we present the recommendations that should be adopted by the optimization research community. Many of these have been suggested in other

\footnotetext{
${ }^{2}$ http://www.mhra.gov.uk/Howweregulate/Medicines/Inspectionandstandards/GoodLaboratoryPractice/Structure/index.htm last accessed 5 Nov 2013
} 
works, including many of those referred to in Section 3.1. However, to our knowledge, there has been very little take up on many of the proposals made and many of the bad practices that were highlighted still exist. We hope that providing an explicit set of recommendations will focus the minds of researchers as to the areas that need to be addressed when proposing a hypothesis and then designing and running simulations to investigate that hypothesis. The recommendations have been split into a number of key areas.

\subsection{Guiding Principles}

There are certain guiding principles that underpin all the other recommendations. This is similar to the guidelines set out by the Committee on Publication Ethics $\left(\mathrm{COPE}^{3}\right)$, which is aimed at journal editors and publishers.

In some sense, they are obvious, but we believe that it is worth stating them explicitly, so that there is no confusion.

\section{Recommendations for GLP4OPT}

R.1 Researchers should adopt the highest ethical standards in conducting their research.

R.2 Researchers should ensure that the work of others is properly cited.

\subsection{Benchmark Datasets}

Some of the papers we reviewed in Section 3.1 were critical of the way that benchmark instances were either introduced into the scientific literature, were biased towards certain algorithms or that randomly generated instances were not a true representation of the problem being considered or that the randomness was biased in some way. Moreover, instances are not always available for other researchers to use and, when randomly generated instances were used the algorithm is not reproducible, meaning that other researchers cannot generate a representative set of instances. Indeed, any generated instance, or its generator, should either be made available to other researchers or the algorithm (including any random number generation) should be defined to enable another researcher to create the same instance.

There are many datasets that have been introduced into the scientific literature. There should be some control as to how a dataset is introduced into the scientific literature, and ultimately utilized.

\section{Recommendations for GLP4OPT}

R.3 Where possible, data should come from the real world, for example, from biological experiments.

R.4 Any benchmark dataset that is accepted into the scientific community should be stored in one central location (see Section 4.16) so that all the datasets are available to all researchers in one place.

\footnotetext{
${ }^{3} \mathrm{http}: / /$ publicationethics.org/
} 
Associated with each instance will be the paper (see R.5) that introduced the benchmark, along with all published results.

R.5 Any dataset that a researcher wants to become a recognized dataset should be subject to the same peer review process as any other scientific paper. Furthermore, where possible, a dataset should be introduced via a stand-alone paper so that the peer review process can focus on the proposed benchmark instances, rather than on an algorithm that is being proposed. The paper should introduce the datasets, and provide all the elements that are set out in R.6 to R.14.

R.6 A benchmark instance should be presented in a standard format, that is specified in such a way that new instances can be presented in the same way. We recognize that this may not be possible for existing datasets, as they may have different formats, but when a new dataset is introduced, the authors should define how future instances should be represented.

R.7 The format in which solutions should be presented should be defined. The format should be amenable to parsing by a computer. We recognize that this may not be possible for existing datasets, as they may have different formats, but when a new dataset is introduced, the authors should define how future instances should be represented.

R.8 When a benchmark instance is proposed, the researcher should provide the necessary means to evaluate each instance. As a minimum a class should be provided in as many languages as possible (e.g. Java, C++, C\#, PHP, Python) which the researcher can utilize to validate the validity and solution quality of any instance that they create. Several examples should be given, to show the evaluation of a number of solutions to help researchers verify that they are evaluating the solutions they produce in the correct way (see R.18).

R.9 When a benchmark instance is proposed, a statistical test should also be suggested that can be used by future researchers to compare against other instances from the benchmark set. This should provide a way to compare different algorithms in a robust way. There are many sources of reference for defining suitable statistical tests. Recent examples include (Derrac et al., 2011) and (García et al., 2010).

R.10 When a benchmark instance is proposed, the researcher should state the recommended computational times used for each instance. Two main timing measures could be adopted. Firstly, the number of times that the evaluation function can be called. Secondly, how 
long the algorithm can run using the same time as returned by recommendation $\mathbf{R . 2 8}$.

R.11 Researchers are encouraged to present a range of different benchmark instances, covering different sizes and complexity. Some of the instances may be solvable to optimality (for example using mathematical programming) so that stochastic methods are able to provide some indication how close they are to optimality. Other instances might be challenging to the methodologies available today and others might be considered to provide challenges for the foreseeable future.

R.12 At the same location (see Section 4.16), where the benchmark instances are located, a history of best known solutions should be available. This, by definition, means that the best known solution is always available.

R.13 Any paper that is published (not just under review) that utilizes one of the standard benchmark datasets must provide ALL the solutions that are referred to in the paper that the authors have published. As an example, if the paper produces 30 solutions for statistical analysis then all those 30 solutions should be available so that other researchers are able to perform comparisons against the set of solutions rather than just the best, mean, median and average (also see R.41). Note, we not suggesting that all the instances in a given dataset have to be reported. A researcher might decide not to use all instances. But, if any instance is used, then ALL the solutions should be made available to the community.

R.14 It is recognized that there are some datasets that have been in the scientific literature for so long that they have become a defacto standard. We cannot simply ignore these and the international advisory board (see Section 6) will define those datasets that should form the basis for this proposed initiative.

\subsection{Non-registered Datasets}

Not every researcher will want to register a benchmark dataset, as defined in Section 4.2. For example, they may not want to wait for a separate paper to be peer reviewed enabling them to claim that they are using a registered dataset. Indeed, they may not be convinced that their dataset will stand up to the scientific review process but they still believe that the dataset is important in the context of their work.

\section{Recommendations for GLP4OPT}

R.15 If a researcher decides not to register a dataset that they use, they should still make the dataset available to the scientific community and should seek to respect recommendations R.6 to R.13. 


\subsection{Presentation of Solutions}

Too often in the optimization research literature, when presenting the results of a simulation the researcher simply reports the value of the evaluation function. If another researcher wants to view the solution, to either verify that the evaluation is correct, or to simply inspect it to give insights for further research, the solution is often impossible to access. Occasionally, results are verified. A good example of this is the Traveling Tournament Problem (TTP) instances, introduced in (Easton et al., 2001). Before a result can be recognized on the web site ${ }^{4}$, the solution has to be sent to Michael Trick, one of the authors who established the benchmarks in (Easton et al., 2001), who will validate that it is correct.

\section{Recommendations for GLP4OPT}

R.16 When presenting the result of a simulation, any solution that is presented must be accompanied by the actual solution in the format that can be validated by R.8. The solutions that are presented (both the solution and its evaluation) should include all those referred to in the paper. For example, any solutions that are used in statistical analysis, or when tuning parameters (see R.41).

\subsection{Evidence of Hybridized Approaches}

Many researchers are now using hybridized approaches to develop effective algorithms. It is important to provide evidence that the hybridized approach is actually effective.

\section{Recommendations for GLP4OPT}

R.17 If a hybridized algorithm is being presented then, unless previously reported in other work, the researchers should provide results from each non-hybridized element, using statistical analysis so that there is evidence that the hybridization is actually effective.

\subsection{Evaluation Function}

The evaluation function is often the most important part of the paper and it is important to get this aspect of the paper correct.

Recommendations for GLP4OPT

R.18 The evaluation function must be described in a way that can be understood and implemented by other researchers.

R.19 Referring to recommendation R.8 it should be possible for another researcher to test their implementation of an evaluation function to check that they have implemented it correctly and that it produces the same values as R.8.

\footnotetext{
${ }^{4}$ http://mat.gsia.cmu.edu/TOURN/
} 


\subsection{Algorithm Presentation}

It is important that an algorithm is presented in a way that another researcher could reproduce it.

\section{Recommendations for GLP4OPT}

R.20 Any algorithm that is presented must be reproducible. This is one of the underpinning tenets of scientific research but some details are often missing, or vague. The optimization research community needs to ensure that all algorithms (and results) are reproducible.

R.21 An algorithm should be presented in pseudo code, rather than a specific programming language. This is to stop any assumptions being made about another researchers' understanding on any given programming language.

R.22 Attention should be given to areas such as variable initialization, how many iterations of certain lines are carried out, all (sub)operations are defined etc.

\subsection{Computational Times}

Recommendations for GLP4OPT Computational times have been an issue for a long time, and will remain so for the foreseeable future. The recommendations in this section, we hope, will resolve many of the issues.

R.23 The researcher should adopt the recommended computational times as provided in R.10, stating which method they are using.

R.24 A researcher is at liberty to use a different computational time, but it must be done in such a way that other researchers are able to use the same timings in future research (see recommendation R.28)

\subsection{Statistics}

The question of which statistics to use is often problematical. However, if recommendation R.9 is adopted then each registered benchmark will come with a set of recommendations as to how different algorithms can be compared. Moreover, this recommendation would have undergone a peer review process so the community will have acknowledged that this statistical test is sufficient. In addition, recommendation R.16 will ensure that there is a sufficient number of solutions available (rather than just the best solution) so that the relevant statistical test has the correct data in order to be applied.

\section{Recommendations for GLP4OPT}

R.25 The necessary statistical test, as defined by R.9, should be applied to the relevant results (e.g. the best known solution and the solutions produced from the current study). 


\subsection{Experimental Conditions}

One of the issues in comparing different algorithms is the environment on which those simulations were run. Whilst we can never account for different programming styles/skills there is a lot we can do to, at least, record the differences in the environment that other researchers will use in the future.

\section{Recommendations for GLP4OPT}

R.26 The full specification of the machine that was used for the experiments should be given. This should include the type of computer, the operating system and version number, the programming language that was used together with the compiler, the amount of memory available to the program, whether parallel processing was employed, if GPUs were utilized etc. Increasingly, researchers are using cloud services. These should also be reported in appropriate detail.

R.27 The date(s) that the experiments were run should be given. This provides another point of reference for future researchers. This is important as the time between running experiments and the paper appearing in print could be a matter of years.

R.28 For each main operating system the community will provide a test program ((Dongarra, 1992) might provide some inspiration for how this can be done) that can be run on a researcher's target machine. This will record the speed of the researcher's machine against some known baseline, informing the researcher how long they should run their algorithm to give a fair comparison with other researchers, particularly the original benchmark. See (McCollum et al., 2010) for an example of this method being used for an international competition.

The benchmark program will also provide a data file that can be stored as part of the paper that is subsequently published which future researchers can refer to in order to compare their algorithm's performance against previous algorithms.

\subsection{Laboratory Notebooks}

As is the case with other scientific disciplines, optimization researchers should be encouraged to maintain a laboratory notebook and make these available to interested parties. Like some of the other recommendations, this is good scientific practice but is noted here so that there is no confusion.

R.29 Optimization researchers should maintain a laboratory notebook. These can be used by the researcher to record their thoughts, ideas and successes/failures and they also provide an important historic record for future researchers to refer to. 
R.30 Researchers are encouraged to make available, via a supplementary file, the relevant parts of their notebooks, once a paper has been published.

R.31 Other researchers should not be critical of notebooks, but should accept them for what they are. A notepad that provides a historic document of the research ideas, thoughts and timeline associated with a given piece of research.

\subsection{Software}

Whether software should be provided as part of the peer review process, and subsequently made available to other researchers, is a moot point in optimization research, although we note that Bioinformatics generally insists on software being made available. There have been recent suggestions that software should be provided. Ince et al. (2012) argue that "anything less than the release of source programs is intolerable for results that depend on computation."

Having access to the software of another researcher would save a lot of time as algorithms would not have to be reimplemented. Moreover, it would remove one area where mistakes could be introduced due to errors/misunderstandings in implementation or the algorithm not being explained well enough to ensure that it can be reproduced.

On the other hand, many researchers are reluctant to provide their code as they might be embarrassed about their coding skills/style and they may feel that they might be asked to explain certain parts of the code or, indeed, provide support.

The fact that an algorithm works now, might not be the case when a new environment is used (e.g new operating systems, different compiler versions etc.). There may also be commercially sensitive reasons why the code should not be in the public domain and researchers may not want to make the code available which has been the result of an investment of weeks/months/years in developing the software.

However, we believe that software which underpins a scientific paper should be part of the peer reviewed scientific archive. This not only adds to the integrity of the discipline but also provides a historical reference that can be utilized by future researchers, perhaps for reasons other than algorithm reproducibility. This might include an historical perspective of programming languages, how coding styles have changed and how the implementations of the same algorithms differ.

\section{Recommendations for GLP4OPT}

R.32 It is beholden on the individual researcher(s) to keep a copy of the software that led to the contribution claimed in a given paper. Researcher(s) should retain an exact copy of the source code, the compiled code and any other environmental factors that are required to reproduce their results. If necessary, they should be able 
to re-run their algorithm and produce exactly the same results (see R.33), even years later.

R.33 In the case of any dispute, authors can be asked to demonstrate their software to show that it produces the results claimed in the paper. This request can only come from the editor-in-chief (or their nominated representative) of the journal to which a paper has been submitted to, or published in.

R.34 The source code for a given paper should be made available, under one of four categories:

1 The source code is made freely available to other researchers

2 The source code is not available to other researchers, but is available to the reviewers who can access the code using the accepted confidentially that is associated with reviewing scientific papers

3 The source code is supplied, but is not made available to either researchers or the reviewers

4 The source code is not made available at all

Unless the first option is chosen, a statement/justification must be given in the paper as to why the source code is being restricted. This statement will be subject to review and may be reason to recommend that the paper not be published.

R.35 Supplying source code does imply that any support will be given to other researchers who later use, or access, the source code.

R.36 The complete environment must be described so that anybody using the source code is able to replicate the computational conditions. As an example, the experimental setup might utilize Matlab, $\mathrm{R}$ or mathematical software. These elements need to be described in a manner that enables reproducibility.

R.37 Source code associated with a published paper should be held in a repository that does not allow updates (any corrections should be done via an erratum) and which can be accessed via a permalink, in the same way that scientific papers are generally available via a DOI. This is to ensure that, like scientific papers, scientific researchers are accessing the same version of the source code as supplied by the authors.

In order to protect the scientific archive, the repository (or repositories) should be maintained by the scientific community, rather than an Open Source repository. 
R.38 Only in exceptional circumstances will access be given to the code without the author's permission, and then only the editor-in-chief of the relevant journal can authorize this. Exceptional circumstances might include accusations of plagiarism, or suspicions of incorrect results being claimed.

R.39 If all the authors have passed away, the software will be available to anybody that requests it, unless other arrangements have been made. This is to allow future researchers to access the code without any hindrance. There is no expectation of support of any kind from colleagues, or institutions.

\subsection{Parameter Selection and Values}

All too often, researchers state that parameter values were set by initial experimentation, arriving at the values used in the paper. This misses many points of interest to the scientific community. For example:

- We are often not told what experiments were carried out to arrive at the values that were used

- We are often not informed how many experiments were run, or how long they took to arrive at the final values

- There is often no statistical analysis, comparing one set of parameters against another in order to demonstrate that a given set of parameter values is better than another or indeed whether the parameters are insensitive to change which could indicate a robust algorithm

- There is often no comparison with samples from outside the tested dataset to provide evidence, or not, that the parameters are robust to problem instances outside of those that are the real focus of the study

\section{Recommendations for GLP4OPT}

R.40 Be specific on how the parameter values were set, detailing the simulations that were carried out, to the point that these should be as reproducible as any other simulations reported in the paper.

R.41 All the solutions that are used to compare the parameters should be made available to other researchers in the same way that the main solutions are also reported (see R.16).

R.42 When making claims that one set of parameter values is better than another, a statistical analysis should support this claim.

R.43 If no evidence is provided to the contrary, no claims should be made that the parameter values that were used for the main simulations presented in the paper are in any way robust, optimal or can be relied upon in other simulations to provide any level of solution quality. 


\subsection{Random Number Generation}

Many papers simply state that a random number is generated, being drawn from a given distribution (e.g. normal, uniform etc.). Not only is the random number generator (RNG) key to reproducibility but the RNG that is used may have certain (sometimes undesirable) properties ((Hellekalek, 1998)). It should be noted that random number generation is applicable to both search methodologies, as well as instance generation.

\section{Recommendations for GLP4OPT}

R.44 The random number generator that was used should be explicitly stated. The coding lines that are used to generate each random variable should be presented, along with any preparatory steps (e.g. setting starting values, seeding the sequence).

R.45 It should be possible for any researcher to generate the same sequence of random numbers, so that the simulation can be reproduced.

\subsection{Termination Criteria}

In many papers it is often stated to carry out a sequence of algorithmic steps until some termination criteria are met. The exact criteria are often obscure or several choices are given and it is not clear which one has been used. For example, "repeat steps $n$ to $m$ until a certain period of time has passed or $q$ iterations have been performed". It should be possible to precisely ascertain the termination criteria that were used for any simulation that is presented.

\section{Recommendations for GLP4OPT}

R.46 Researchers should precisely define the termination criteria for any algorithm that is presented.

\subsection{GLP4OPT Adherence and Support}

Evidence to demonstrate compliance with GLP4OPT should be provided as a supplementary file(s) to the main article. This enables authors to show compliance, without having to take up valuable space in the main article. In line with current practice, any supplementary files are subject to peer review.

If the recommendations in this paper are embraced by the community, this might lead to a web presence for GLP4OPT, which could reference, and link to, all the papers that have been peer reviewed in-line with the recommendations contained in this paper.

There may also be an opportunity for one of the Operations Research societies to lead on the GLP4OPT standard but we recognise that this is not a decision that is within the scope of this article and would be subject to much more discussion. 
In the remainder of this section, we provide specific recommendations as to what should constitute a supplementary file(s) and how a web site GLP4OPT might support the community in the future. Section 6 provides more thoughts as to how GLP4OPT might be progressed.

\section{Recommendations for GLP4OPT}

R.47 Any benchmarks that are introduced (see R.5 to R.14) into the scientific literature must be recorded in the journal's supplementary repository (see R.54).

R.48 The GLP4OPT supplementary file must define which version of the standard has been used. As mentioned in the Introduction, the version introduced here is GLP4OPT version 1.00.

R.49 If a journal or publisher has subscribed to GLP4OPT then it should insist on a supplementary file that reviewers can access to check against compliance with the standard. It should also be made available to those readers that are enable to access the main paper.

R.50 Once (and if) established, the GLP4OPT web site will maintain the best known solutions for each registered dataset, along with a history of best known solutions (see R.12).

R.51 ALL solutions that are referred to in a given paper must be uploaded to the journal as a supplementary file(s) (see R.13). This includes any solutions used to establish parameter settings (see R.41)

R.52 Once (and if) established, the GLP4OPT web site will contain a repository of all the papers that have used a given registered benchmark dataset.

R.53 Once (and if) established, the GLP4OPT web site will maintain the set of historical datasets (see (R.14), including best known solutions and all papers that have used those datasets.

R.54 It is recognized that many of the data specified in this section might be held as a supplementary file on the journal's we site. In this case, the GLP4OPT web site will contain the necessary links to the supplementary data.

\section{$5 \quad$ Reviewing against GLP4OPT}

If the GLP4OPT standard was adopted, it would lead naturally to a set of prescribed questions that any reviewer might ask, and which authors might be expected to respond to. 
Of course, a negative answer to any of these questions would not automatically disqualify the paper from being published. That decision still ultimately lies with the reviewers and the editors, but it might help provide additional focus/information for the review process. Moreover, knowing that the reviewers will explicitly look at these aspects of the paper may help the authors in planning their experiments and the reporting of their work.

\section{Progression}

In this section, we outline several ideas as to how the views expressed in this paper could be progressed in order to provide the optimization research community with a more robust experimental environment.

In our view, it is the lack of a progression plan that has hindered anything happening until now. The ideas that have been expressed since the 1970's have all been very valid proposals but it was left to the community to adopt them. This has not happened, or only to a very limited extent.

To progress the adoption of the proposals outlined in this paper, we suggest the following, once this paper has been published.

1. Publishers and journals are approached, asking them to adopt the standards and requesting that they incorporate the standard into the work flow, as well as informing authors, reviewers and editors that the standard has been adopted. This would be similar to them adopting COPE (Committee on Publication Ethics)

2. Operations Research societies are approached asking that they adopt this standard, and promote its use to it members

3. Funding agencies are approached to ask if they would be willing to adopt GLP4OPT, specifying that their use is a condition of any grant that they award

4. The views/opinions of the entire community (including the industrial community) should be sought to ask their ideas/feedback on the suggested GLP4OPT standards. This will lead to a new version of GLP4OPT

5. Operations Research societies are approached to ask if one of them would like to take the lead on maintaining, developing and promoting GLP4OPT

6. A Management Board could be established that oversees GLP4OPT, in the same way that OECD acts for Good Laboratory Practice for nonclinical research. The Management Board will be responsible for (but not limited to) raising funds, maintaining and developing GLP4OPT, liaising with the community (authors, readers, publishers, societies etc.), ensuring that GLP4OPT is adopted etc.

The Management Board should be international in make up, should include experienced and early career researchers, as well as the industrial 
community. The Management Board must also represent all of optimization research.

7. An International Advisory Board could be established. The make up, and remit, of this board will be the responsibility of GLP4OPT's Management Board but it should certainly be international in nature and should provide coverage across all of optimization research, and have representation from experienced and early career researchers as well as the industrial community.

8. A web site could be established to support GLP4OPT.

None of the authors of this paper are suggesting that, as authors of the paper, that they should serve in any particular role with regard to GLP4OPT. If this paper is published, the authors will progress GLP4OPT until suitable structures have been established.

\section{Conclusions}

This paper provides the opportunity for those that embrace the ideas outlined in this paper (or those that are developed as a result of this paper) to work to a common set of standards which are recognized across the disciplines. Even though they may not be perfect, and subject to future enhancements, at least we will all be working to the same set of imperfect standards, rather than everybody working to a different set.

We realize that papers such as these are not without controversy and that there will never be a set of guiding principles that everybody agrees to and, of course, any research group or individual has the right to simply ignore any recommendations that arise as a result of this paper. But, we hope that, if any of the principles outlined in this paper are adopted, the community would recognize the potential benefit both in terms of carrying out and reporting its research but also providing training to its researchers in carrying out high quality research.

We also hope that adherence to the GLP4OPT standards, would give other researchers some level of confidence as the quality of the research that has been conducted but it would also provide future researchers with a set of data that will enable them to more easily compare against their own research. Moreover, it would build up a valuable set of data that could be used in a variety of ways in the future, above and beyond, simply using the benchmark data to compare against a current research idea.

\section{References}

Adenso-Diaz, B., M. Laguna. 2006. Fine-tuning of algorithms using fractional experimental designs and local search. Operations Research 54(1) 99-114. doi:10.1287/opre.1050.0243. 
Ahuja, R.V., J. Orlin. 1996. Use of representative operation counts in computational testing of algorithms. INFORMS Journal on Computing 8(3) 318-330. doi:10.1287/ijoc.8.3.318.

Anon. 2013. Announcement: Reducing our irreproducibility. Nature 496(7446) 398. doi:10.1038/496398a.

Archetti, C., M.G. Speranza. 2008. The Split Delivery Vehicle Routing Problem: A Survey. Golden, B and Raghavan, S and Wasil, E, ed., Vehicle Routing Problem: Latest Advances and New Challanges, Operations Research Computer Science Interfaces, vol. 43. Springer US, 103-122. doi:10.1007/978-0387-77778-8_5.

Baker, M. 2012. Independent labs to verify high-profile papers: Reproducibility initiative aims to speed up preclinical research. Nature (News), 14 Aug 2012 doi:10.1038/nature.2012.11176.

Balinski, M.L. 1978. On the reporting of computational experiments. Mathematical Programming 15(1) 315. doi:10.1007/BF01609035.

Barr, R.S., B.L. Golden, J.P. Kelly, M.G.C. Resende, W.R. Stewart Jr. 1995. Designing and reporting on computational experiments with heuristic methods. Journal of Heuristics 1(1) 9-32. doi:10.1007/BF02430363.

Begley, C. G., L. M. Ellis. 2012. Drug development: Raise standards for preclinical cancer research. Nature 483(7391) 531-533. doi:10.1038/483531a.

Bellmore, M., G.L. Nemhauser. 1968. Travelling salesman problem: A survey. Operations Research 16(3) 538-558. doi:10.1287/opre.16.3.538.

Błazewicz, J., W. Domschke, E. Pesch. 1996. The job shop scheduling problem: Conventional and new solution techniques. European Journal of Operational Research 93(1) 1-33. doi:10.1016/0377-2217(95)00362-2.

Błazewicz, J., K.H. Ecker, E. Pesch, G. Schmidt, J. Weglarz. 2007. Handbook on Scheduling. Springer.

Błazewicz, J., P. Formanowicz, M. Kasprzak. 2005. Selected combinatorial problems of computational biology. European Journal of Operational Research 161(3) 585-597. doi:10.1016/j.ejor.2003.10.054.

Boylan, J. E., P. Goodwin, M. Mohammadipour, A. A. Syntetos. 2015. Reproducibility in forecasting research. International Journal of Forecasting 31(1) 79 - 90. doi:http://dx.doi.org/10.1016/j.ijforecast.2014.05.008.

Braysy, O., W. Dullaert, M. Gendreau. 2004. Evolutionary algorithms for the vehicle routing problem with time windows. Journal of Heuristics 10(6) 587611. doi:10.1007/s10732-005-5431-6. 
Braysy, O., M. Gendreau. 2005a. Vehicle routing problem with time windows, part I: Route construction and local search algorithms. Transportation Science 39(1) 104-118. doi:10.1287/trsc.1030.0056.

Braysy, O., M. Gendreau. 2005b. Vehicle routing problem with time windows, part II: Metaheuristics. Transportation Science 39(1) 119-139. doi: $10.1287 / \operatorname{trsc} .1030 .0057$.

Brown, L. 1999. Technical and Military Imperatives: A Radar History of World War 2. CRC Press.

Brucker, P., Yu N. Sotskov, F. Werner. 2007. Complexity of shop-scheduling problems with fixed number of jobs: a survey. Mathematical Methods of Operations Research 65(3) 461-481. doi:10.1007/s00186-006-0127-8.

Burkard, R.E., V.G. Deineko, R. Van Dal, J.A.A. Van der Veen, G.J. Woeginger. 1998. Well-solvable special cases of the traveling salesman problem: A survey. SIAM Review 40(3) 496-546. doi:10.1137/S0036144596297514.

Burke, E. K., M. Gendreau, M. Hyde, G. Kendall, G. Ochoa, E. Özcan, R. Qu. 2013. Hyper-heuristics: a survey of the state of the art. Journal of the Operational Research Society 64(12) 1695-1724. doi:10.1057/jors.2013.71.

Cattrysse, DG, L.N. Van Wassenhove. 1992. A survey of algorithms for the generalized assignment problem. European Journal of Operational Research 60(3) 260-272. doi:10.1016/0377-2217(92)90077-M.

Cheng, R.W., M. Gen, Y. Tsujimura. 1999a. A tutorial survey of jobshop scheduling problems using genetic algorithms, part II: Hybrid genetic search strategies. Computers \& Industrial Engineering 36(2) 343-364. doi: 10.1016/S0360-8352(99)00136-9.

Cheng, R.W., M. Gen, Y. Tsujimura. 1999b. A tutorial survey of jobshop scheduling problems using genetic algorithms: Part II. Hybrid genetic search strategies. Computers \& Industrial Engineering 37(1-2) 51-55. doi: 10.1016/S0360-8352(99)00022-4.

Cook, W.J. 2011. In Pursuit of the Traveling Salesman: Mathematics at the Limits of Computation. Princeton University Press.

Cordeau, J.F., M. Gendreau, G. Laporte, J.Y.Potvin, F. Semet. 2002. A guide to vehicle routing heuristics. Journal of the Operational Research Society 53(5) 512-522. doi:10.1057/palgrave/jors/2601319.

Coy, S.P., B.L. Golden B.L., G.C. Runger, E.A. Wasil. 2001. Using experimental design to find effective parameter settings for heuristics. Journal of Heuristics 7(1) 77-97. doi:10.1023/A:1026569813391.

Crowder, H., R.S. Dembo, J.M. Mulvey. 1979. On reporting computational experiments with mathematical software. ACM Transactions on Mathematical Software 5(2) 193-203. doi:10.1145/355826.355833. 
Crowder, H.P., R.S. Dembo, J.M. Mulvey. 1978. Reporting computational experiments in mathematical programming. Mathematical Programming 15(1) 316-329. doi:10.1007/BF01609036.

Derrac, J., S. García, D. Molina, F. Herrera. 2011. A practical tutorial on the use of nonparametric statistical tests as a methodology for comparing evolutionary and swarm intelligence algorithms. Swarm and Evolutionary Computation 1(1) 3-18. doi:10.1016/j.swevo.2011.02.002.

Doerner, K.F., V. Schmid. 2010. Survey: Matheuristics for Rich Vehicle Routing Problems. Blesa, MJ and Blum, C and Raidl, G and Roli, A and Sampels, M, ed., Hybrid Metaheuristics, Lecture Notes in Computer Science, vol. 6373. 206-221. 7th International Workshop on Hybrid Metaheuristics, Vienna, Austria, Oct 01-02, 2010.

Dongarra, J.J. 1992. Performance of various computers using standard linear equations software. ACM SIGARCH Computer Architecture News 20(3) 2244. doi:10.1145/141868.141871.

Easley, R.W., C.S. Madden, M.G. Dunn. 2000. Conducting marketing science: The role of replication in the research process. Journal of Business Research 48(1) 83 - 92. doi:http://dx.doi.org/10.1016/S0148-2963(98)00079-4.

Easton, K., G. Nemhauser, M. Trick. 2001. The Traveling Tournament Problem Description and Benchmarks, chap. Principles and Practice of Constraint Programming CP 2001: 7th International Conference, CP 2001 Paphos, Cyprus, November 26 December 1, 2001 Proceedings. Lecture Notes in Computer Science 2239, Springer Berlin Heidelberg, 580-584. doi:10.1007/3$540-45578-7 \_43$.

Ernst, A.T., H. Jiang, M. Krishnamoorthy, D. Sier. 2004. Staff scheduling and rostering: A review of applications, methods and models. European Journal of Operational Research 153(1) 3-27. doi:10.1016/S0377-2217(03)00095-X.

Evanschitzky, H., J.S. Armstrong. 2010. Replications of forecasting research. International Journal of Forecasting 26(1) 4-8. doi: http://dx.doi.org/10.1016/j.ijforecast.2009.09.003.

Evanschitzky, H., C. Baumgarth, R. Hubbard, J. S. Armstrong. 2007. Replication research's disturbing trend. Journal of Business Research 60(4) 411-415. doi:http://dx.doi.org/10.1016/j.jbusres.2006.12.003.

Fildes, R. 1979. Quantitative forecastingThe state of the art: Extrapolative models. Journal of the Operational Research Society 30(8) 691-710. doi: 10.1057/jors.1979.172.

Fildes, R. 1985. Quantitative forecastingThe state of the art: Econometric models. Journal of the Operational Research Society 30(7) 549-580. doi: 10.1057/jors.1985.99. 
Fildes, R., K. Nikolopoulos, S.F. Crone, A.A. Syntetos. 2008. Forecasting and operational research: a review. Journal of the Operational Research Society 59(9) 1150-1172. doi:10.1057/palgrave.jors.2602597.

García, S., A. Fernández, J. Luengo, F. Herrera. 2010. Advanced nonparametric tests for multiple comparisons in the design of experiments in computational intelligence and data mining: Experimental analysis of power. Information Sciences 180(10) 2044-2064. doi:10.1016/j.ins.2009.12.010.

Gass, S.I., A.A. Assad. 2006. An Annotated Timeline of Operations Research: An Informal History. Springer.

Gendreau, M., J-Y. Potvin, O. Braysy, G. Hasle, A. Lokketangen. 2008. Metaheuristics for the Vehicle Routing Problem and Its Extensions: A Categorized Bibliography. Golden, B and Raghavan, S and Wasil, E, ed., Vehicle Routing Problem: Latest Advances and New Challenges, Operations Research Computer Science Interfaces, vol. 43. Springer US, 143-169. doi:10.1007/978-0387-77778-8_7.

Ghobbar, A.A., C.H. Friend. 2003. Evaluation of forecasting methods for intermittent parts demand in the field of aviation: a predictive model. Computers 83 Operations Research 30(14) 2097 - $2114 . \quad$ doi: http://dx.doi.org/10.1016/S0305-0548(02)00125-9.

Golden, B.L., A.A. Assad, W.A. Wasil, E. Baker. 1986. Experimentation in optimization. European Journal of Operational Research 27(1) 1-16. doi: 10.1016/S0377-2217(86)80002-9.

Golden, B.L., W.R. Stewart. 1985. The Traveling Salesman Problem, chap. Empirical Analysis of Heuristics. John Wiley \& Sons Ltd., 207-249.

Greenberg, H.J. 1990. Computational testing: Why, how and how much. ORSA Journal on Computing 2(1) 94-97. doi:10.1287/ijoc.2.1.94.

Hellekalek, P. 1998. Good random number generators are (not so) easy to find. Mathematics and Computers in Simulation 46(5-6) 485-505. doi: 10.1016/S0378-4754(98)00078-0.

Hoffman, A., M. Mannos, D. Sokolowsky, N. Wiegmann. 1953. Computational experience in solving linear programs. Journal of the Society for Industrial and Applied Mathematics 1(1) 17-33.

Hooker, J.N. 1994. Needed: An empirical science of algorithms. Operations Research 42(2) 201-212. doi:10.1287/opre.42.2.201.

Hooker, J.N. 1995. Testing heuristics - we have it all wrong. Journal of Heuristics 1(1) 33-42. doi:10.1007/BF02430364.

Hooker, J.N. 2007. Good and bad futures for constraint programming (and operations research). Constraint Programming Letters 1 21-32. 
Hubbard, R., J.S. Armstrong. 1994. Replications and extensions in marketing: Rarely published but quite contrary. International Journal of Research in Marketing 11(3) 233 - 248. doi:http://dx.doi.org/10.1016/01678116(94)90003-5.

Hubbard, R., D.E. Vetter. 1996. An empirical comparison of published replication research in accounting, economics, finance, management, and marketing. Journal of Business Research 35(2) 153 - 164 . doi: http://dx.doi.org/10.1016/0148-2963(95)00084-4.

Ignizio, J.P. 1971. On the establishment of standards for comparing algorithm performance. Interfaces 2(1) 8-11. doi:10.1287/inte.2.1.8.

Ince, D. C., L. Hatton, J. Graham-Cumming. 2012. The case for open computer programs. Nature 482(7386) 485-488. doi:10.1038/nature10836.

Ioannidis, J.A. 2005. Contradicted and initially stronger effects in highly cited clinical research. Journal of the American Medical Association 294(2) 218228. doi:10.1001/jama.294.2.218.

Jackson, R.H.F., P.T. Boggs, S.G. Nash, S. Powell. 1991. Guidelines for reporting results of computational experiments. report of the ad hoc committee. Mathematical Programming 49(1-3) 413-425. doi:10.1007/BF01588801.

Jackson, R.H.F., J.M. Mulvey. 1978. A critical review of methods for comparing mathematical programming algorithms and software (1953-1977). Journal of Research of the National Bureau of Standards 83(6) 563-584. doi: 10.6028/jres.083.038.

Jaillet, P., M.R. Wagner. 2008. Online Vehicle Routing Problems: A Survey. Golden, B and Raghavan, S and Wasil, E, ed., Vehicle Routing Problem: Latest Advances and New Challenges, Operations Research Computer Science Interfaces, vol. 43. Springer US, 221-237. doi:10.1007/978-0-387-77778-8_10.

Jourdan, L., M. Basseur, E.-G. Talbi. 2009. Hybridizing exact methods and metaheuristics: A taxonomy. European Journal of Operational Research 199(3) 620-629. doi:10.1016/j.ejor.2007.07.035.

Kendall, G., S. Knust, C.C. Ribeiro, S S. Urrutia. 2010. Scheduling in sports: An annotated bibliography. Computers 83 Operations Research 37(1) 1-19. doi:10.1016/j.cor.2009.05.013.

Kiran, A.S., M.L. Smith. 1984. Simulation studies in job shop scheduling - 1. A survey. Computers 83 Industrial Engineering 8(2) 87-93. doi:10.1016/03608352(84)90001-9.

Kirkpatrick, S., C.D. Gelatt Jr., M. Vecchi. 1983. Optimization by simulated annealing. Science 220(4598) 671-680. doi:10.1126/science.220.4598.671. 
Laporte, G. 2009. Fifty years of bvehicle routing. Transportation Science 43(4) 408-416. doi:10.1287/trsc.1090.0301.

Lee, C-Y., J. Bard, M. Pinedo, W.E. Wilhelm. 1993. Guidelines for reporting computational resuts in iie transactions. IIE Transactions 25(6) 121-123. doi:10.1080/07408179308964335.

Lin, E.Y.H. 1998. A bibliographical survey on some well-known non-standard knapsack problems. INFOR 36(4) 274-317.

Loiola, E.M., N.M.M. de Abreu, P.O. Boaventura-Netto, P. Hahn, T. Querido. 2007. A survey for the quadratic assignment problem. European Journal of Operational Research 176(2) 657-690. doi:10.1016/j.ejor.2005.09.032.

Lukasiak, P., J. Błazewicz, M. Milostan. 2010. Some operations research methods for analyzing protein sequences and structures. Annals of Operations Research 175(1) 9-35. doi:10.1007/s10479-009-0652-y.

Lust, T., J. Teghem. 2010. The multiobjective traveling salesman problem: A survey and a new approach. C.A.C Coello, C. Dhaenens, L. Jourdan, eds., Studies in Computational Intelligence, vol. 272. Springer-Verlag Berlin, 119141.

Lust, T., J. Teghem. 2012. The multiobjective multidimensional knapsack problem: a survey and a new approach. International Transactions in Operational Research 19(4) 495-520. doi:10.1111/j.1475-3995.2011.00840.x.

Marshall, E. 1983. The murky world of toxicity testing. Science 220(4602) 1130-1132. doi:10.1126/science.6857237.

McCollum, B., A. Schaerf, B. Paechter, P. McMullan, R. Lewis, A.J. Parkes, L. Di Gaspero, R. Qu, E. K. Burke. 2010. Setting the research agenda in automated timetabling: The second international timetabling competition. INFORMS Journal on Computing 22(1) 120-130. doi:10.1287/ijoc.1090.0320.

McGeoch, C.C. 1996. Toward an experimental method for algorithm simulation. INFORMS Journal on Computing 8(1) 1-15. doi:10.1287/ijoc.8.1.1.

Miller, D. M., D. Williams. 2003. Shrinkage estimators of time series seasonal factors and their effect on forecasting accuracy. International Journal of Forecasting 19(4) 669 - 684. doi:http://dx.doi.org/10.1016/S0169-2070(02)000778.

Miller, H.E. 1976. Personnel scheduling in public systems - A survey. Socio-Economic Planning Sciences 10(6) 241-249. doi:10.1016/00380121(76)90011-2.

Pentico, D.W. 2007. Assignment problems: A golden anniversary survey. European Journal of Operational Research 176(2) 774-793. doi: 10.1016/j.ejor.2005.09.014. 
Pisinger, D. 2007. The quadratic knapsack problem - A survey. Discrete Applied Mathematics 155(5) 623-648. doi:10.1016/j.dam.2006.08.007.

Prinz, F., T. Schlange, K. Asadullah. 2011. Believe it or not: how much can we rely on published data on potential drug targets? Nature Reviews Drug Discovery 10(7391) 10-11. doi:10.1038/nrd3439-c1.

Rardin, R.L., R. Uzsoy. 2001. Experimental evaluation of heuristic optimization algorithms: a tutorial. Journal of Heuristics 7(3) 261-304. doi: 10.1023/A:1011319115230.

Rasmussen, R.V., M.A. Trick. 2008. Round robin scheduling - a survey. European Journal of Operational Research 188(3) 617-636. doi: 10.1016/j.ejor.2007.05.046.

Russell, J. F. 2013. If a job is worth doing, it is worth doing twice. Nature 496(7443) 7. doi:10.1038/496007a.

Salkin, H.M., C.A.D. Kluyver. 1975. Knapsack problem - survey. Naval Research Logistics 22(1) 127-144. doi:10.1002/nav.3800220110.

Sörensen, K. 2015. Metaheuristics - the metaphor exposed. International Transactions in Operational Research 22(1) 3-18. doi:10.1111/itor.12001.

Sörensen, K., F. Glover. 2013. Encyclopedia of Operations Research and Management Science, chap. Metaheuristics. 3rd ed. Springer.

Taillard, É.D., P. Waelti, J. Zuber. 2008. Few statistical tests for proportions comparison. European Journal of Operational Research 185(3) 1336-1350. doi:10.1016/j.ejor.2006.03.070.

Talbi, E-G. 2009. Metaheuristics: From Design to Implementation. Wiley.

Trapero, J. R., N. Kourentzes, R. Fildes. 2015. Identification of sales forecasting models. Journal of the Operational Research Society 66(2) 299-307. doi: $10.1057 /$ jors.2013.174.

Vaux, D. L. 2012. Research methods: Know when your numbers are significant. Nature 492(7428) 180-181. doi:10.1038/492180a.

Vidal, T., T.G. Crainic, M. Gendreau, C. Prins. 2013. Heuristics for multiattribute vehicle routing problems: A survey and synthesis. European Journal of Operational Research 231(1) 1-21. doi:10.1016/j.ejor.2013.02.053.

Vines, T.H., A.Y.K. Albert, R.L. Andrew, F. Débarre, D.G. Bock, M.T. Franklin, K.J. Gilbert, J-S. Moore, S. Renaut, D.J. Rennison. 2013. The availability of research data declines rapidly with article age. Current Biology 24(1) 94-97. doi:10.1016/j.cub.2013.11.014. 
Wilbaut, C., S. Hanafi, S. Salhi. 2008. A survey of effective heuristics and their application to a variety of knapsack problems. IMA Journal of Management Mathematics 19(3) 227-244. doi:10.1093/imaman/dpn004.

Zanakis, S. H., J.R. Evans, A.A. Vazacopoulos. 1989. Heuristic methods and applications: A categorized survey. European Journal of Operational Research 43(1) 88-110. doi:10.1016/0377-2217(89)90412-8. 